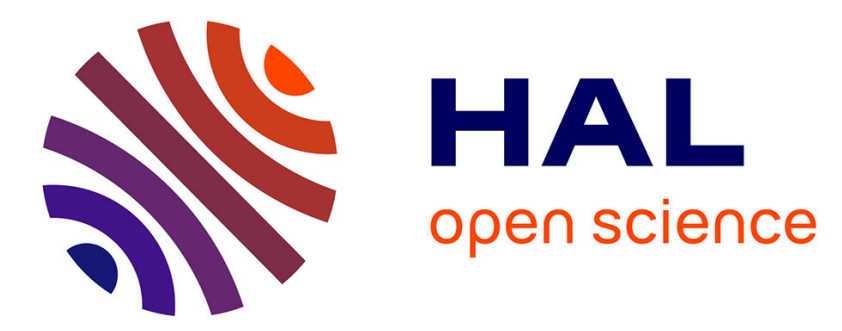

\title{
General form of consensus optimization for distributed OPF in HVAC-VSC-HVDC systems
}

\author{
Monica Aragues-Penalba, Tung Lam Nguyen, Raphael Caire, Andreas \\ Sumper, Samuel Galceran-Arellano, Quoc-Tuan Tran
}

\section{To cite this version:}

Monica Aragues-Penalba, Tung Lam Nguyen, Raphael Caire, Andreas Sumper, Samuel GalceranArellano, et al.. General form of consensus optimization for distributed OPF in HVAC-VSCHVDC systems. International Journal of Electrical Power \& Energy Systems, 2020, 121, pp.106049. 10.1016/j.ijepes.2020.106049 . hal-03260239

\section{HAL Id: hal-03260239 \\ https://hal.science/hal-03260239}

Submitted on 20 Jan 2022

HAL is a multi-disciplinary open access archive for the deposit and dissemination of scientific research documents, whether they are published or not. The documents may come from teaching and research institutions in France or abroad, or from public or private research centers.
L'archive ouverte pluridisciplinaire HAL, est destinée au dépôt et à la diffusion de documents scientifiques de niveau recherche, publiés ou non, émanant des établissements d'enseignement et de recherche français ou étrangers, des laboratoires publics ou privés. 


\title{
General form of consensus optimization for distributed OPF in HVAC-VSC-HVDC systems
}

\author{
Mònica Aragüés-Peñalba ${ }^{a, *}$, Tung Lam Nguyen ${ }^{b}$, Raphael Caire ${ }^{\mathrm{b}}$, Andreas \\ Sumper $^{\mathrm{a}}$, Samuel Galceran-Arellano ${ }^{\mathrm{a}}$, Quoc-Tuan Tran ${ }^{\mathrm{b}}$ \\ ${ }^{a}$ Centre d'Innovació Tecnològica en Convertidors Estàtics i Accionaments \\ (CITCEA-UPC), Departament d'Enginyeria Elèctrica, Universitat Politècnica de \\ Catalunya. ETS d'Enginyeria Industrial de Barcelona, Av. Diagonal, 647, 2nd floor, \\ 08028 Barcelona, Spain. \\ ${ }^{b}$ Grenoble Electrical Engineering Laboratory (G2Elab), 21 Avenue des Martyrs, 38000 \\ Grenoble, France \\ ${ }^{c}$ Alternative Energies and Atomic Energy Commission (CEA), National Institute for \\ Solar Energy (INES), F-73375 Le Bourget-du-Lac, France
}

\begin{abstract}
In this paper a distributed algorithm for ensuring Optimal Power Flows in hybrid AC-DC systems is proposed. While centralized OPF formulations require information of many variables of all the network nodes, the present approach relies on solving an OPF locally in each AC agent and AC-DC agent that only requires to exchange the voltages corresponding to adjacent nodes. Therefore, operation schemes based on this approach would be specially interesting when communications with the central unit fail. This algorithm is applied to a $3 \mathrm{DC}-5 \mathrm{AC}$ bus system, showing that the obtained results coincide with those of the centralized formulation. Additionally, the dynamic behavior of this system operated under the proposed algorithm is analysed in a real-time platform in a realistic communication environment.
\end{abstract}

Keywords: Hybrid AC-DC systems, OPF, Distributed optimization, ADMM

\footnotetext{
*Corresponding author

Email address: monica.aragues@citcea.upc.edu (Mònica Aragüés-Peñalba)
} 


\section{Introduction}

The evolution of power electronics and the increasing integration of renewable energy sources into power systems is guiding their planning, operation and control. At transmission system level, High Voltage Alternating Current (HVAC) is being combined with High Voltage Direct Current (HVDC) to form hybrid networks, due to the technical and economic limitations of HVAC -and benefits of HVDC- in bulk power transmission over long distances, submarine power transmission and interconnection of asynchronous systems. As shown in [1], DC networks can be operated to provide support to AC systems, increasing its security of supply and resilience in case of outages.

For steady-state analysis, system planning and security, the power flows in these AC-DC systems need to be determined [2]. In [3] an open-source software (MATACDC) is presented to analyse the steady state interactions between HVAC and HVDC grids. To ensure the optimal operation of hybrid HVDC-HVAC systems, Optimal Power Flows (OPF) formulations are being investigated with several objective functions. In [4] a two-stage solution approach for solving the problem of multiobjective OPF in hybrid AC/DC grids with HVDC is proposed based on the application of multiobjective evolutionary algorithms. It focuses on the coordination of the economy, voltage deviation, and environmental benefits for hybrid AC/DC grids. Most of the proposals addressing $\mathrm{OPF}$ in hybrid $\mathrm{AC} / \mathrm{DC}$ grids focus on a single objective function and are based on a centralized approach [5, 6]. In [5], an algorithm to optimally operate HVAC-HVDC systems is proposed and combined with voltage droop control of grid side Voltage Source Converters (VSC). In [6] a relaxed mathematical model for solving OPF for AC-DC grids is developed to guarantee convergence and global optimality. Centralized approaches rely on the existing communication systems, already available for monitoring and control purposes. In these cases, a central unit collects information of all the nodes and branches, determines the new operational state and sends the appropriate references to each node.

Although offering a global vision of the network, the central approach might be difficult to scale, requires the permanent healthy state of the central computation unit and of all the communication links. Furthermore, it assumes that the operators accept to share their data with the central unit. An OPF based on a distributed approach could overcome these challenges. The potential benefits identified in distributed algorithms include the share

of limited amount of information between a subset of the nodes, the cyberse- 
curity enhancement, the lower expense in the communication infrastructure and the computational superiority versus centralized algorithms thanks to the parallelization of computations [7].

In [8], a comparison between different distributed optimization is presented. One of the distributed optimization techniques, based on the Augmented Lagrangian decomposition, is the ADMM (Alternate Direction Method of Multipliers) [9]. It has been proposed for solving distributed OPF in AC grids in many studies, for instance in [10-13]. One of the first studies to propose a distributed algorithm for the optimal operation of $\mathrm{AC}$ systems is [10], based on a region-based optimization process for AC systems where limited information is exchanged between neighbouring regions. In [11], an ADMM based algorithm is proposed for OPF in AC grids using sequential convex approximations. In [12] the AC grid equations are simplified through a DC formulation and solved in a distributed manner through ADMM. The authors from [13] propose a distributed algorithm in which each generator is considered an agent for ensuring optimal reactive power control in power systems. ADMM has been applied to perform OPF in DC grids in [14], where a distributed secondary control for multiterminal DC grids is proposed to operate the DC system optimally after solving a Quadratrically Constrained Quadratic Program (QCQP).

Nevertheless, the distributed operation of hybrid AC-DC systems has been briefly analyzed. In [15], the $\mathrm{AC}$ power flow equations are simplified with a DC linear approach and converter losses are not taken into consideration. In [16] the complete non-linear formulation for the AC grid is used. The approach is based on separating the whole grid in subsystems and a high amount of information exchange inside the nodes of each region or subsystem is required (voltages, angles, active and reactive powers from generators and converters). Moreover, the system behaviour under this operation scheme in the time domain is not addressed. Recently, in [17], an ADMM problem is formulated to solve an optimization that determines a smooth operation point for hourly dispatches in multiterminal AC-DC grids. Converters are assumed to be lossless and the transient behavior of the system is not addressed. The authors from [18] propose a synchronous ADMM approach for the operation of $\mathrm{AC} / \mathrm{DC}$ systems based on the use of the average value of boundary variables of adjacent regions. The network is partitioned in based on transmission lines. Converter losses are not taken into consideration. Despite the distributed approach reaches the same results that the centralized one, convergence problems are identified and still need to be solved. Most 
of the few studies addressing the distributed operation of $\mathrm{AC} / \mathrm{DC}$ systems based on ADMM do not take into consideration the power converter losses and all the existing ones focus on the steady state analysis, without deeping into the operation in the time domain.

To the best knowledge of the authors, there is no study that covers the distributed optimal operation of hybrid AC-DC systems and analyses how the system behaves dynamically in a real communication environment. The present paper proposes a novel formulation for the tertiary control of HVACHVDC systems, where a distributed optimal operation for minimum losses (transmission and converter losses) of AC-DC systems is developed based on ADMM and on a multi-agent system approach [19]. The optimization problem is separated in smaller subproblems, each of which is solved locally by an agent of the network (AC agent or AC-DC agent). By simulating a case study of $3 \mathrm{DC}-5 \mathrm{AC}$ bus network, it is shown that the distributed approach reaches the same optimal operation point as the centralized scheme. Then, the distributed implementation of this case study is tested in an experimental setup that runs the power system with demand changes in real time through OPAL-RT. The data from the physical system is exchanged with the outside environment through an external switch and via user data protocol. The communication among agents (Raspberrys $\mathrm{Pi}$ ) is in a client/server manner with gRPC protocol. Even if the communication delays among agents are different, they cooperate with their neighbours and achieve the optimal operation of the system.

The main contributions of this study are: i) the formulation of a distributed tertiary control for AC-DC systems where only communications between adjacent nodes are required (Section 3), ii) only voltage information needs to be exchanged between these nodes (Section 3), iii) the formulation considers power converter losses and is extendable to any AC-DC grid topology regardless if it contains meshes or not and to other objective functions (Section 3), iv) it is proven that AC-DC systems can optimally operate under the proposed algorithm after showing stable response in an experimental setup running in real time (Sections 4 and 5).

Centralized approaches for OPFs in hybrid AC/DC systems require that all the data of the system are collected by a central control. This implies drawbacks including the reliability and security vulnerability of the central unit as a common point of failure, the high computation time in the central unit for large power systems and high communication infrastructure needs due to the geographical extension of the power system [20]. The proposed dis- 
tributed approach avoids the use of a central unit and reduces the communication infrastructure used as well as the amount of information to be shared. Thanks to the parallelization of computations, each agent solves locally a problem and with the appropriate coordination with adjacent neighbours, and only exchanging voltage information, the system reaches the optimum operation. The present paper validates its application in HVAC-VSC-HVDC systems. However, the approach can also be applied for hybrid AC-DC systems at other voltage levels, for instance integrating microgrids.

The paper is structured as follows. Section 2 explains the General form of consensus optimization. Then, a formulation for OPF in AC-DC systems is presented in Section 3. This formulation enables to propose a distributed approach for OPF in AC-DC systems based on ADMM in Section 3.2. Section 4 applies the distributed OPF developed to a 3 DC- 5 AC network and validates the results obtained with the centralized OPF approach. Section 5 analyses the transient behavior of the previous hybrid AC-DC system in a real communication environment [21]. Finally, in Section 6, conclusions are drawn.

\section{General form of consensus optimization}

The ADMM algorithm is based on a decomposition-coordination procedure [9], in which the solutions to small local subproblems are coordinated to find a solution to a large global problem. This algorithm benefits from the dual decomposition and augmented Lagrangian methods for constrained optimization.

Let's assume a network of size $N$ that can be represented by a connected graph, is composed of $K$ subsystems of size $N_{k}$, being each one managed by an agent. Each agent can only communicate with its neighbouring agents (and therefore with subsystems that are directly connected to the subsystem it manages). The general consensus problem can be formulated as the sum of all the local objective functions $f_{k}$ handled by each agent:

$$
\min _{x} \sum_{k=1}^{K} f_{k}\left(x_{k}\right)
$$

subject to

$$
x_{k}-z_{k}=0, \quad k=1, \ldots, K
$$


where $x_{k}$ is the vector of local variables of subsystem $k$ such that $x \in \mathbb{R}^{N_{k}}$ and consists of a selection of the components of the global variable $z \in \mathbb{R}^{N}$. Each agent will be in charge of optimising its objective function and on agreeing (reaching consensus) on the local variable components that map to the same global variable components. To indicate that the local variable component $\left(x_{k}\right)_{j}$ corresponds to the global variable component $\left(z_{k}\right)_{j}$, the following index notation is used: $g=\mathcal{G}(k, j)$. Therefore, the consensus between local and global variables is reached when:

$$
\left(x_{k}\right)_{j}=z_{\mathcal{G}(k, j)}, \quad k=1, \ldots, K, j=1, \ldots, N_{k}
$$

Other equality and inequality constraints can be added to coupling Equation (2), as reflected in [9].

The stated optimization problem can be solved using ADMM through the augmented Lagrangian in Equation (4) and through the iterative process reflected in Equations (5),(6),(7), where $\rho>0$ is the penalty parameter and $\lambda$ is the Lagrange multiplier.

$$
\begin{gathered}
L_{\rho}(x, z, \lambda)=\sum_{k=1}^{K}\left(f_{k}\left(x_{k}\right)+\lambda_{k}^{T}\left(x_{k}-z_{k}\right)+\frac{\rho}{2}\left\|x_{k}-z_{k}\right\|_{2}^{2}\right) \\
x_{k}^{i+1}:=\underset{x_{k}}{\operatorname{argmin}}\left(f_{k}\left(x_{k}\right)+\left(\lambda_{k}^{i}\right)^{T} x_{k}+\frac{\rho}{2}\left\|x_{k}-z_{k}^{i}\right\|_{2}^{2}\right) \\
z^{i+1}:=\underset{z}{\operatorname{argmin}}\left(\sum_{k=1}^{N}\left(-\left(\lambda_{k}^{i}\right)^{T} z_{k}+\frac{\rho}{2}\left\|x_{k}^{i+1}-z_{k}\right\|_{2}^{2}\right)\right) \\
\lambda_{k}^{i+1}:=\lambda_{k}^{i}+\rho\left(x_{k}^{i+1}-z_{k}^{i+1}\right)
\end{gathered}
$$

The update of each local variable (Equation (5)) and of the Lagrange multiplier (Equation (7)) can be performed in parallel for each agent $\mathrm{k}$. The global variable update (Equation (6)) is done decoupling the components of $z$ :

$$
z_{g}^{i+1}:=\frac{\sum_{g=\mathcal{G}(k, j)}\left(\left(x_{k}^{i+1}\right)_{j}+\frac{1}{\rho}\left(\lambda_{k}^{i}\right)_{j}\right)}{\sum_{g=\mathcal{G}(k, j)} 1}
$$

In fact, the global variable update can be obtained by a local averaging of $z_{g}$ components, as shown in Equation (10) knowing that after the first iteration 
$(i=1)$ :

$$
\begin{gathered}
\sum_{g=\mathcal{G}(k, j)}\left(\lambda_{k}^{i}\right)_{j}=0 \\
z_{g}^{i+1}:=\frac{1}{n_{g}} \sum_{g=\mathcal{G}_{(k, j)}}\left(x_{k}^{i+1}\right)_{j}
\end{gathered}
$$

where $n_{g}$ is the number of local variable entries that correspond to the global variable entry $z_{g}$.

\section{Optimal power flow}

We consider a hybrid AC-DC electrical network that consists of a set of AC nodes: $\mathcal{N}_{a c}=\left\{1,2, \ldots, N_{a c}\right\}$ and a set of DC nodes: $\mathcal{N}_{d c}=\left\{1,2, \ldots, N_{d c}\right\}$. The formulation for AC networks power flows is presented in detail in [20] and summarised in Section 6.1. It is extended for DC networks in Section 6.2 .

To enable the application of ADMM to AC/DC systems power flow optimisation, different agents are identified taking into account that each agent can only communicate with its neighbours (and consequently with subsystems that are directly connected to the subsystem it manages). Therefore, two type of AC nodes or agents can be identified:

- $A C$ agent: AC node that is not connected to the DC grid. It has information of its own $\mathrm{AC}$ bus and of the adjacent $\mathrm{AC}$ buses to which it is connected.

- $A C$-DC agent: $\mathrm{AC}$ node that is connected to the $\mathrm{DC}$ grid. It has information of its own $\mathrm{AC}$ and $\mathrm{DC}$ bus, of the the adjacent $\mathrm{AC}$ buses to which it is connected and of the adjacent DC buses to which it is connected.

Based on the formulation detailed in Section 6, the active power and reactive power balance at bus $k$ is defined by Equations (11) and (12), respectively, while power in DC nodes can be expressed as reflected in Equation (13):

$$
\begin{aligned}
p_{k} & =\hat{\mathbf{v}}_{k}^{T} \cdot \mathbf{z}_{k}^{p} \cdot \hat{\mathbf{v}}_{k} \\
q_{k} & =\hat{\mathbf{v}}_{k}^{T} \cdot \mathbf{z}_{k}^{q} \cdot \hat{\mathbf{v}}_{k} \\
p_{l, d c} & =\hat{\mathbf{v}}_{k}^{T} \cdot \mathbf{z}_{l}^{d c} \cdot \hat{\mathbf{v}}_{k}
\end{aligned}
$$




\section{Nomenclature}

$\hat{\mathbf{v}} \quad$ Vector of local variables for agent in bus $k$

$\lambda_{k} \quad$ Lagrange multiplier of subsystem $k$

$\mathcal{G}_{\text {gen }} \quad$ Set of buses containing a generator

$\mathcal{N}_{a c} \quad$ Set of AC nodes

$\mathcal{N}_{d c} \quad$ Set of DC nodes

$\rho \quad$ Penalty parameter

$\mathbf{z}_{g, k} \quad$ Vector of global variables for agent in bus $k$

$B \quad$ Susceptance matrix of the AC grid

$G \quad$ Conductance matrix of the AC grid

$G_{d c} \quad$ Admittance matrix of the DC grid

$i_{k} \quad$ Injected current at bus $k$

$i_{k}^{i m} \quad$ Imaginary part of the injected current at bus $k$

$i_{k}^{r e} \quad$ Real part of the injected current at bus $k$

$P_{k, v s c}^{\max }$ Active power upper limit of VSC in $k$

$P_{k, v s c}^{\min }$ Active power lower limit of VSC in $k$

$p_{k} \quad$ Active power at bus $k$

$p_{k}^{L} \quad$ Active power of the load L connected at bus $k$
$P_{k}^{\max }$ Active power upper limit of generator in $k$

$P_{k}^{\min } \quad$ Active power lower limit of generator in $k$

$p_{v s c l o s s, k}$ Converter losses of VSC in bus $k$

$Q_{k, v s c}^{\max }$ Reactive power upper limit of VSC in $k$

$Q_{k, v s c}^{\min }$ Reactive power lower limit of VSC in $k$

$q_{k} \quad$ Reactive power at bus $k$

$q_{k}^{L} \quad$ Reactive power of the load L connected at bus $k$

$Q_{k}^{\max }$ Reactive power upper limit of generator in $k$

$Q_{k}^{\text {min }} \quad$ Reactive power lower limit of generator in $k$

$S_{k, v s c}^{\max }$ Apparent power limit of VSC in node $k$

$s_{k} \quad$ Injected apparent power at bus $k$

$v_{k} \quad$ Voltage at bus $k$

$v_{k}^{i m} \quad$ Imaginary part of the voltage at bus $k$

$v_{k}^{\max } \quad$ Upper limit of voltage at bus $k$

$v_{k}^{\min } \quad$ Lower limit of voltage at bus $k$

$v_{k}^{r e} \quad$ Real part of the voltage at bus $k$

$Y \quad$ Admittance matrix of the AC grid 


$$
\begin{gathered}
\hat{\mathbf{v}}=\left[\begin{array}{c}
\mathbf{v}^{r e} \\
\mathbf{v}^{i m} \\
\mathbf{v}_{d c}
\end{array}\right] \\
\mathbf{z}^{p}=\left[\begin{array}{ccc}
\mathbf{G} & -\mathbf{B} & \mathbf{0} \\
\mathbf{B} & \mathbf{G} & \mathbf{0} \\
\mathbf{0} & \mathbf{0} & \mathbf{0}
\end{array}\right] \\
\mathbf{z}^{q}=\left[\begin{array}{ccc}
-\mathbf{B} & -\mathbf{G} & \mathbf{0} \\
\mathbf{G} & -\mathbf{B} & \mathbf{0} \\
\mathbf{0} & \mathbf{0} & \mathbf{0}
\end{array}\right] \\
\mathbf{z}^{d c}=\left[\begin{array}{ccc}
\mathbf{0} & \mathbf{0} & \mathbf{0} \\
\mathbf{0} & \mathbf{0} & \mathbf{0} \\
\mathbf{0} & \mathbf{0} & \mathbf{G}_{d c}
\end{array}\right] \\
\mathbf{z}_{k}^{p}=\left[\begin{array}{c}
\mathbf{0} \\
\mathbf{z}^{p}(k,:) \\
\mathbf{0} \\
\mathbf{z}^{p}\left(k+N_{a c},:\right) \\
\mathbf{0}
\end{array}\right] \\
\mathbf{z}_{k}^{q}=\left[\begin{array}{c}
\mathbf{0} \\
\mathbf{z}^{q}(k,:) \\
\mathbf{0} \\
\mathbf{z}^{q}\left(k+N_{a c},:\right) \\
\mathbf{0}
\end{array}\right] \\
z_{l}^{d c}=\left[\begin{array}{c}
\mathbf{0} \\
\mathbf{z}^{d c}\left(2 N_{a c}+l,:\right) \\
\mathbf{0}
\end{array}\right]
\end{gathered}
$$

\section{AC agents}

For those AC nodes that are not connected to the DC grid, the following equations need to be satisfied.

If the $\mathrm{AC}$ node has generator and load, then:

$$
\begin{aligned}
& P_{k}^{\text {min }} \leq \hat{\mathbf{v}}_{k}^{T} \cdot \mathbf{z}_{k}^{p} \cdot \hat{\mathbf{v}}_{k}+p_{k}^{L} \leq P_{k}^{\text {max }}, \quad k \in \mathcal{N}_{a c}, k \in \mathcal{G}_{g e n} \\
& Q_{k}^{\text {min }} \leq \hat{\mathbf{v}}_{k}^{T} \cdot \mathbf{z}_{k}^{q} \cdot \hat{\mathbf{v}}_{k}+q_{k}^{L} \leq Q_{k}^{\text {max }}, \quad k \in \mathcal{N}_{a c}, k \in \mathcal{G}_{\text {gen }}
\end{aligned}
$$


where $P_{k}^{\min }$ and $P_{k}^{\max }$ represent the active power lower and upper limits of generator in $k, Q_{k}^{\min }$ and $Q_{k}^{\max }$ represent the reactive power lower and upper limits of generator in $k$. If the AC node only has load, then:

$$
\begin{aligned}
& \hat{\mathbf{v}}_{k}^{T} \cdot \mathbf{z}_{k}^{p} \cdot \hat{\mathbf{v}}_{k}+p_{k}^{L}=0, \quad k \in \mathcal{N}_{a c}, k \notin \mathcal{G}_{g e n} \\
& \hat{\mathbf{v}}_{k}^{T} \cdot \mathbf{z}_{k}^{q} \cdot \hat{\mathbf{v}}_{k}+q_{k}^{L}=0, \quad k \in \mathcal{N}_{a c}, k \notin \mathcal{G}_{g e n}
\end{aligned}
$$

In all the cases, voltage limits need to be satisfied for the $\mathrm{AC}$ nodes:

$$
\left(\mathbf{v}_{k}^{\min }\right)^{2} \leq\left(\mathbf{v}_{k}^{r e}\right)^{2}+\left(v_{k}^{i m}\right)^{2} \leq\left(\mathbf{v}_{k}^{\max }\right)^{2}, \quad k \in \mathcal{N}_{a c}
$$

\section{AC-DC agents}

VSC technology allows the independent control of active and reactive power. The VSC control modes are mainly active power control or DC voltage control and reactive power control or AC voltage control. The choice of the control modes depends on the application of the VSC-HVDC and on the type of the AC grids to which they are connected [22]. However, a VSC responsible for DC voltage control is required to ensure the power balance between the sending and receiving converters. The control modes do not affect the problem formulation (the number and type of variables involved in each agent remain the same), but the number of VSCs does, at is directly related with the number of $\mathrm{AC} / \mathrm{DC}$ agents of the system. For those $\mathrm{AC}$ nodes that are connected to the $\mathrm{DC}$ grid through a $\mathrm{VSC}$, the following equations need to be satisfied.

If the AC node has generator and load, then:

$$
\begin{aligned}
P_{k}^{\text {min }} \leq & \hat{\mathbf{v}}_{k}^{T} \cdot \mathbf{z}_{k}^{p} \cdot \hat{\mathbf{v}}_{k}+\mathbf{v}_{k}^{T} \cdot \mathbf{z}_{l}^{d c} \cdot \hat{\mathbf{v}}_{k} \pm p_{\text {vscloss }, k}+p_{k}^{L} \leq P_{k}^{\max }, \quad k \in \mathcal{N}_{a c}, k \in \mathcal{G}_{\text {gen }} \\
& P_{k, \text { vsc }}^{\text {min }} \leq \mathbf{v}_{k}^{T} \cdot \mathbf{z}_{l}^{d c} \cdot \hat{\mathbf{v}}_{k} \pm p_{\text {vscloss }, k} \leq P_{k, \text { vsc }}^{\text {max }}, \quad k \in \mathcal{N}_{a c}, k \in \mathcal{G}_{\text {gen }}
\end{aligned}
$$

where $p_{v s c l o s s, k}$ represent converter losses, modeled according to a second order polynomial and distinguishing rectifier and inverter operation [23].

$$
Q_{k}^{\min }+Q_{k, v s c}^{\min } \leq \hat{\mathbf{v}}_{k}^{T} \cdot \mathbf{z}_{k}^{q} \cdot \hat{\mathbf{v}}_{k}+q_{k}^{L} \leq Q_{k}^{\max }+Q_{k, v s c}^{\max }, \quad k \in \mathcal{N}_{a c}, k \in \mathcal{G}_{\text {gen }}
$$

Additionally, the operation inside the power limits of the converter needs to be ensured:

$$
\left(\hat{\mathbf{v}}_{k}^{T} \cdot \mathbf{z}_{l}^{d c} \cdot \hat{\mathbf{v}}_{k} \pm p_{\text {vscloss }, k}\right)^{2}+\left(Q_{k}^{\max }-\left(\hat{\mathbf{v}}_{k}^{T} \cdot \mathbf{z}_{k}^{q} \cdot \hat{\mathbf{v}}_{k}+q_{k}^{L}\right)\right)^{2} \leq\left(S_{k, v s c}^{\max }\right)^{2}
$$


If the $\mathrm{AC}$ node only has load, then: If the $\mathrm{AC}$ node only has load, then:

$$
\begin{gathered}
\hat{\mathbf{v}}_{k}^{T} \cdot \mathbf{z}_{k}^{p} \cdot \hat{\mathbf{v}}_{k}+\mathbf{v}_{k}^{T} \cdot \mathbf{z}_{l}^{d c} \cdot \hat{\mathbf{v}}_{k} \pm p_{\text {vscloss }, k}+p_{k}^{L}=0, \quad k \in \mathcal{N}_{a c}, k \notin \mathcal{G}_{g e n} \\
Q_{k, v s c}^{\text {min }} \leq \hat{\mathbf{v}}_{k}^{T} \cdot \mathbf{z}_{k}^{q} \cdot \hat{\mathbf{v}}_{k}+q_{k}^{L} \leq Q_{k, v s c}^{\max }, \quad k \in \mathcal{N}_{a c}, k \notin \mathcal{G}_{g e n} \\
\left(\hat{\mathbf{v}}_{k}^{T} \cdot \mathbf{z}_{l}^{d c} \cdot \hat{\mathbf{v}}_{k} \pm p_{\text {vscloss }, k}\right)^{2}+\left(\hat{\mathbf{v}}_{k}^{T} \cdot \mathbf{z}_{k}^{q} \cdot \hat{\mathbf{v}}_{k}+q_{k}^{L}\right)^{2} \leq\left(S_{k, v s c}^{\text {max }}\right)^{2}, \quad k \in \mathcal{N}_{a c}, k \notin \mathcal{G}_{\text {gen }}
\end{gathered}
$$

In all the cases, voltage limits need to be satisfied for $\mathrm{AC}$ nodes and $\mathrm{DC}$ nodes:

$$
\begin{gathered}
\left(\mathbf{v}_{k}^{\min }\right)^{2} \leq\left(\mathbf{v}_{k}^{r e}\right)^{2}+\left(v_{k}^{i m}\right)^{2} \leq\left(\mathbf{v}_{k}^{\max }\right)^{2}, \quad k \in \mathcal{N}_{a c} \\
\mathbf{v}_{l, d c}^{\min } \leq \mathbf{v}_{l, d c} \leq \mathbf{v}_{l, d c}^{\max }, \quad l \in \mathcal{N}_{d c}
\end{gathered}
$$

As example, the construction of the local vector $\hat{\mathbf{v}}$ for each agent of the ACDC system depicted in Figure 1 is reflected in Figure 2. In this case, there are two AC agents (in buses 2 and 3) and two AC-DC agents (in bus 1 and bus 4 ). The variables constituting the local vector of each agent are detailed in Figure 2, as well as their relation with the global variables.

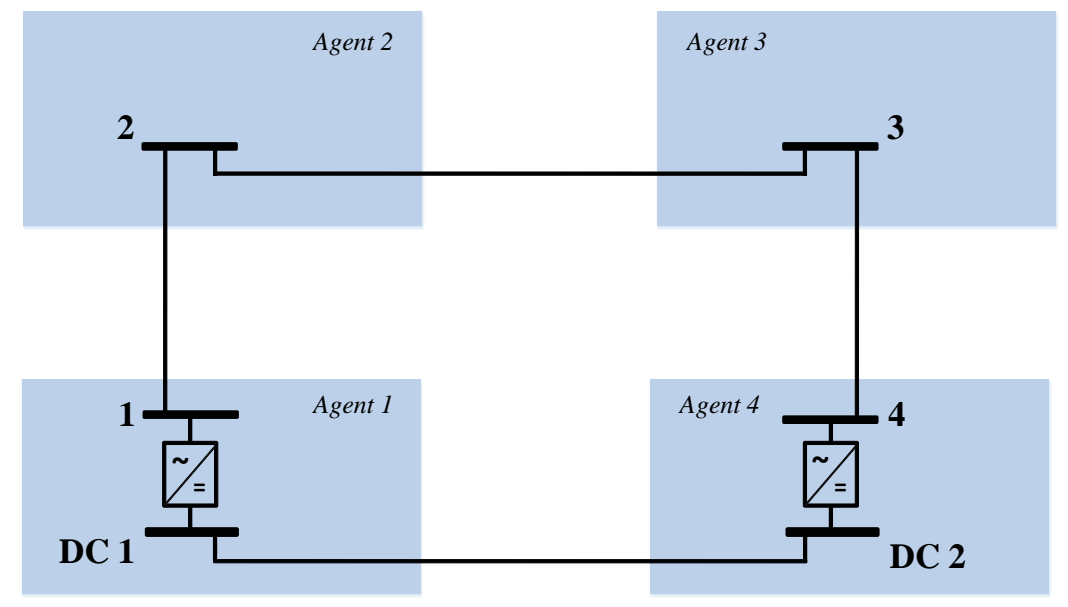

Figure 1: Agents identification in a 4 AC 2 DC bus example network

\section{Objective function}

The objective function here analysed is the loss minimization over all the AC-DC grid, which is formulated as follows:

$$
\min _{\hat{\mathbf{v}}} \hat{\mathbf{v}}^{T} \cdot \mathbf{z}^{p} \cdot \hat{\mathbf{v}}+\hat{\mathbf{v}}^{T} \cdot \mathbf{z}^{d c} \cdot \hat{\mathbf{v}}
$$




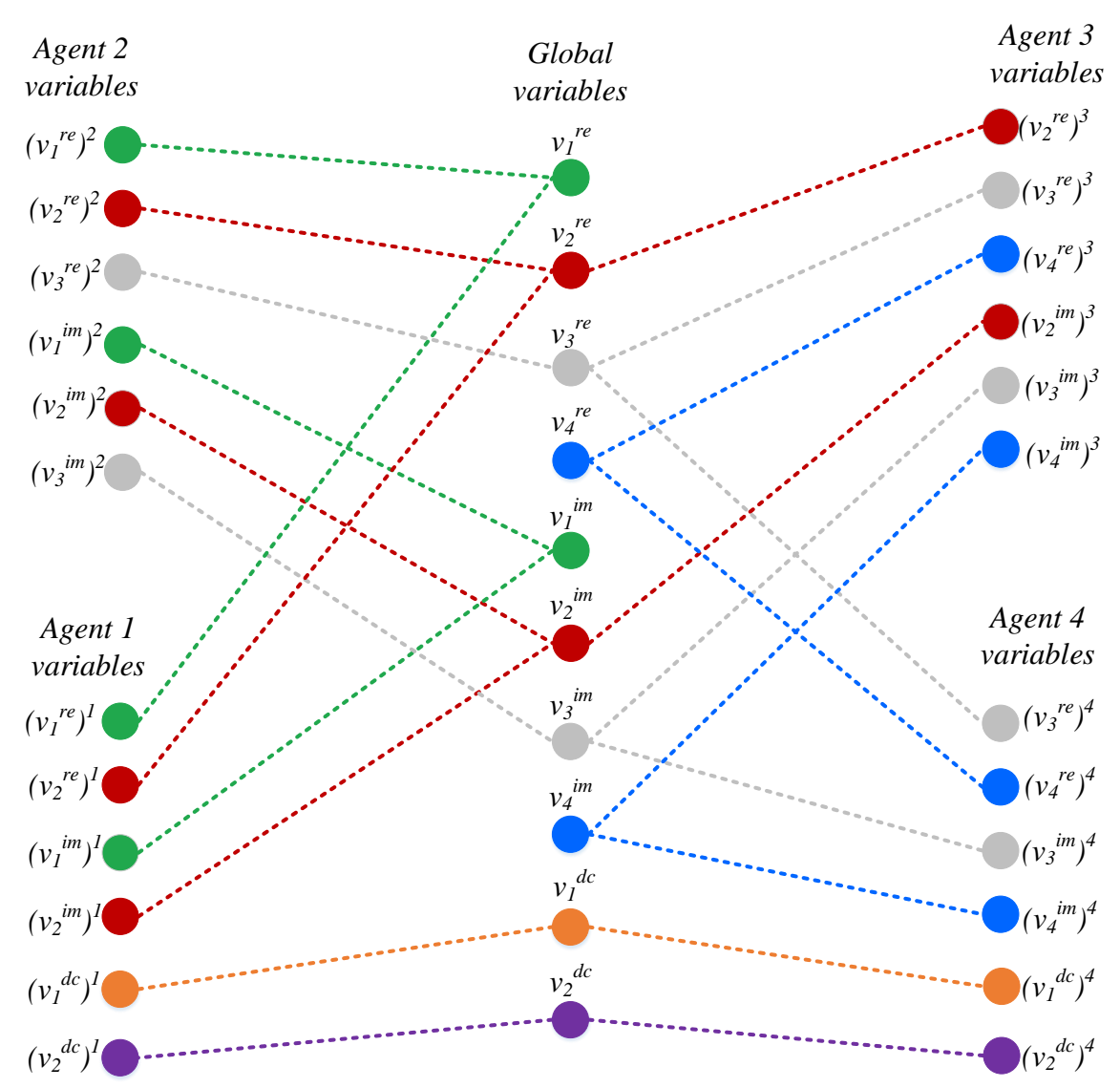

Figure 2: Local vectors construction for system in Figure 1

For the case of minimization of power system losses, the higher is the number of VSCs, higher are the losses from converter stations, but also there is higher flexibility for power flows. Other objective functions could be considered, for instance minimum operation costs for the whole system or minimum deviation from a preset voltage profile.

\subsection{Centralized $A C-D C$ optimal power flow}

The centralized formulation of the AC-DC OPF for loss minimization corresponds to the optimization problem with the objective function reflected in Equation (28) subject to the set of constraints in Equations from (14) to (27). 


\subsection{Distributed AC-DC optimal power flow}

The optimization problem in Section 3.1 can be separated in smaller subproblems, each of which will be solved locally by an agent of the network (either an AC agent or an AC-DC agent). The algorithm that deals with the solution of each subproblem and leads to the solution of the whole optimization problem is based on ADMM (see Section 2) and presented in Algorithm 1. It has been implemented in Matlab.

\section{Case study}

The centralized and distributed AC-DC optimal power flow formulations described in Sections 3.1 and 3.2, implemented in Matlab, have been applied to a particular $3 \mathrm{DC}-5 \mathrm{AC}$ system, which is based on the IEEE 5 bus test system and has also been used in [6,23]. The network is illustrated in Figure 3. The DC and AC systems are linked through three VSC converters. The power in the $\mathrm{DC}$ network is injected into the $\mathrm{AC}$ grid through the connected inverters, which are responsible for the DC grid voltage control and which provide reactive power support to the $\mathrm{AC}$ grid when needed. The $\mathrm{AC}$ grid has two generators, one in bus 1 and another in bus 2. Loads are connected to buses 2, 3, 4 and 5. The DC grid is connected in AC buses 2, 3, and 5 . The power converters rating is $50 \mathrm{MVA}$ and bus 1 is assumed to be the slack. The active and reactive power demand in the $\mathrm{AC}$ buses are given by vectors $p_{L}$ and $q_{L}$, respectively: $p^{L}=[0,20,45,40,60] \mathrm{MW}, q^{L}=[0,10,15,5,10]$ MVAr.

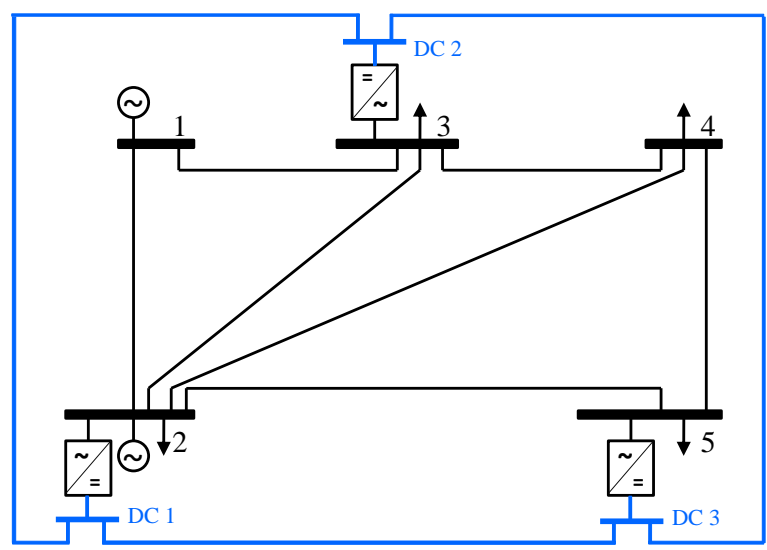

Figure 3: 3 DC - 5 AC bus system 


\section{Algorithm 1 ADMM for distributed AC-DC OPF at agent $k$}

1: $i=1: \mathbf{z}_{g, k} \leftarrow \mathbf{z}_{0}, \boldsymbol{\lambda}_{k} \leftarrow \boldsymbol{\lambda}_{0} \quad \triangleright$ at first iteration, give initial guess value of global variables and Lagrangian multipliers

2: repeat

$\triangleright$ at each iteration $i$, the agent solves the local problem:

- if agent $k$ is a $A C$ agent:

$$
\min _{\hat{\mathbf{v}}_{k}} \quad \hat{\mathbf{v}}_{k}^{T} \cdot \mathbf{z}_{k}^{p} \cdot \hat{\mathbf{v}}_{k}+\lambda_{k}^{T} \hat{\mathbf{v}}_{k}+\frac{\rho}{2}\left\|\hat{\mathbf{v}}_{k}-\mathbf{z}_{g, k}\right\|_{2}^{2}
$$

subject to:

$$
\left(\mathbf{v}_{k}^{\min }\right)^{2} \leq\left(\mathbf{v}_{k}^{r e}\right)^{2}+\left(v_{k}^{i m}\right)^{2} \leq\left(\mathbf{v}_{k}^{\max }\right)^{2}, \quad k \in \mathcal{N}_{a c}
$$

$\forall k \in \mathcal{G}_{\text {gen }}:$

$$
\begin{aligned}
P_{k}^{\text {min }} & \leq \hat{\mathbf{v}}_{k}^{T} \cdot \mathbf{z}_{k}^{p} \cdot \hat{\mathbf{v}}_{k}+p_{k}^{L} \leq P_{k}^{\text {max }} \\
Q_{k}^{\text {min }} & \leq \hat{\mathbf{v}}_{k}^{T} \cdot \mathbf{z}_{k}^{q} \cdot \hat{\mathbf{v}}_{k}+q_{k}^{L} \leq Q_{k}^{\text {max }}
\end{aligned}
$$

$\forall k \notin \mathcal{G}_{g e n}:$

$$
\begin{aligned}
& \hat{\mathbf{v}}_{k}^{T} \cdot \mathbf{z}_{k}^{p} \cdot \hat{\mathbf{v}}_{k}+p_{k}^{L}=0 \\
& \hat{\mathbf{v}}_{k}^{T} \cdot \mathbf{z}_{k}^{q} \cdot \hat{\mathbf{v}}_{k}+q_{k}^{L}=0
\end{aligned}
$$

- if agent $k$ is a $A C-D C$ agent:

$$
\min _{\hat{\mathbf{v}}_{k}} \quad \hat{\mathbf{v}}_{k}^{T} \cdot \mathbf{z}_{k}^{p} \cdot \hat{\mathbf{v}}_{k}+\hat{\mathbf{v}}_{k}^{T} \cdot \mathbf{z}_{l}^{d c} \cdot \hat{\mathbf{v}}_{k}+\lambda_{k}^{T} \hat{\mathbf{v}}_{k}+\frac{\rho}{2}\left\|\hat{\mathbf{v}}_{k}-\mathbf{z}_{g, k}\right\|_{2}^{2}
$$

subject to:

$$
\begin{aligned}
& \left(\mathbf{v}_{k}^{\min }\right)^{2} \leq\left(\mathbf{v}_{k}^{r e}\right)^{2}+\left(v_{k}^{i m}\right)^{2} \leq\left(\mathbf{v}_{k}^{\max }\right)^{2}, \quad k \in \mathcal{N}_{a c} \\
& \mathbf{v}_{l, d c}^{\min } \leq \mathbf{v}_{l, d c} \leq \mathbf{v}_{l, d c}^{\max } \quad l \in \mathcal{N}_{d c}
\end{aligned}
$$

$\forall k \in \mathcal{G}_{g e n}:$

$$
\begin{aligned}
& P_{k}^{\text {min }} \leq \hat{\mathbf{v}}_{k}^{T} \cdot \mathbf{z}_{k}^{p} \cdot \hat{\mathbf{v}}_{k}+\mathbf{v}_{k}^{T} \cdot \mathbf{z}_{l}^{d c} \cdot \hat{\mathbf{v}}_{k} \\
& \quad \pm p_{v s c l o s s, k}+p_{k}^{L} \leq P_{k}^{\text {max }} \\
& P_{k, v s c}^{\text {min }} \leq \mathbf{v}_{k}^{T} \cdot \mathbf{z}_{l}^{d c} \cdot \hat{\mathbf{v}}_{k} \pm p_{v s c l o s s, k} \leq P_{k, v s c}^{\max } \\
& Q_{k}^{\text {min }}+Q_{k, v s c}^{\text {min }} \leq \hat{\mathbf{v}}_{k}^{T} \cdot \mathbf{z}_{k}^{q} \cdot \hat{\mathbf{v}}_{k}+q_{k}^{L} \leq Q_{k}^{\text {max }}+Q_{k, v s c}^{\max } \\
& \left(\hat{\mathbf{v}}_{k}^{T} \cdot \mathbf{z}_{l}^{d c} \cdot \hat{\mathbf{v}}_{k} \pm p_{v s c l o s s, k}\right)^{2} \\
& \quad+\left(\hat{\mathbf{v}}_{k}^{T} \cdot \mathbf{z}_{k}^{q} \cdot \hat{\mathbf{v}}_{k}+q_{k}^{L}\right)^{2} \leq\left(S_{k, v s c}^{\max }\right)^{2}
\end{aligned}
$$

$\forall k \notin \mathcal{G}_{g e n}:$

$$
\begin{aligned}
& \hat{\mathbf{v}}_{k}^{T} \cdot \mathbf{z}_{k}^{p} \cdot \hat{\mathbf{v}}_{k}+\mathbf{v}_{k}^{T} \cdot \mathbf{z}_{l}^{d c} \cdot \hat{\mathbf{v}}_{k} \pm p_{\text {vscloss }, k}+p_{k}^{L}=0 \\
& Q_{k, \text { vsc }}^{\min } \leq \hat{\mathbf{v}}_{k}^{T} \cdot \mathbf{z}_{k}^{q} \cdot \hat{\mathbf{v}}_{k}+q_{k}^{L} \leq Q_{k, v s c}^{\max } \\
& \left(\hat{\mathbf{v}}_{k}^{T} \cdot \mathbf{z}_{l}^{d c} \cdot \hat{\mathbf{v}}_{k} \pm p_{\text {vscloss }, k}\right)^{2} \\
& \quad+\left(Q_{k}^{\max }-\left(\hat{\mathbf{v}}_{k}^{T} \cdot \mathbf{z}_{k}^{q} \cdot \hat{\mathbf{v}}_{k}+q_{k}^{L}\right)\right)^{2} \leq\left(S_{k, v s c}^{\max }\right)^{2}
\end{aligned}
$$

3: $\quad \hat{\mathbf{v}}_{k}^{i} \leftarrow \hat{\mathbf{v}}_{k}$

4: $\quad z_{g, k}^{i}:=\frac{\left.\sum_{g=\mathcal{G}(k, j)}\left(\hat{\mathbf{v}}_{k}^{i}\right)_{j}+\frac{1}{\rho}\left(\lambda_{k}^{i-1}\right)_{j}\right)}{\sum_{g=\mathcal{G}(k, j)} 1} \quad 14 \quad \triangleright$ global variable update for each agent

$5: \quad \lambda_{k}^{i}=\lambda_{k}^{i-1}+\rho\left(\hat{\mathbf{v}}_{k}^{i}-\mathbf{z}_{g, k}^{i}\right)$

6: $\quad i \leftarrow i+1$

$\triangleright$ Lagrange multiplier update for each agent $\triangleright$ move to next iteration 

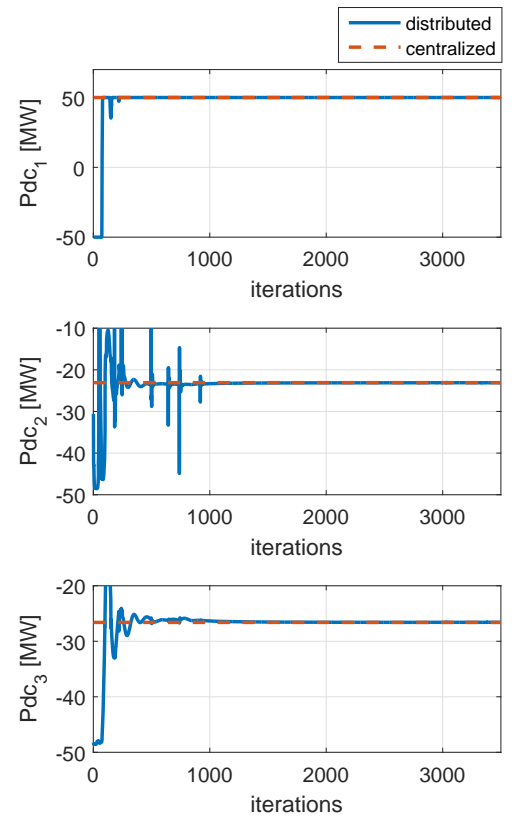

(a) Power injected to the buses of the DC system
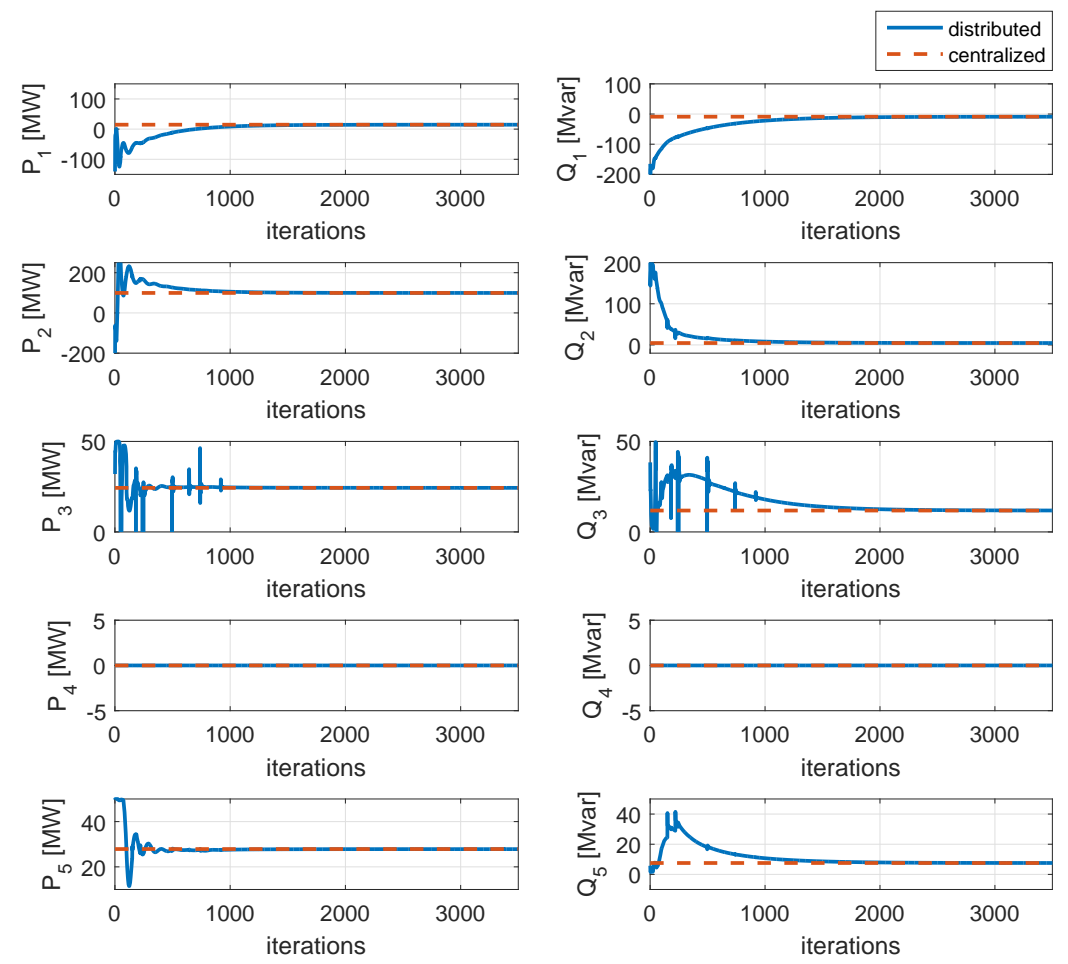

15

(b) Active and reactive power injected to the buses of the AC system

Figure 4: Power injected to DC buses and AC buses of the system 
The DC and AC voltages obtained in the different buses for loss minimization are reflected in Table 1. The minimum losses for the analyzed situation are 0.0159 p.u. (1.59 MW). The active and reactive powers injected in the $\mathrm{AC}$ buses are depicted in Figure 4b. The active power injected in the DC buses are shown in Figure 4a. It is shown that after 3500 iterations, the consensus value is reached. This figure also shows how the results obtained from the distributed formulation (continuous blue line) are the same as the results obtained with a centralized formulation (dashed orange line).

Table 1: Bus voltages for loss minimisation

\begin{tabular}{|c|ccc|}
\hline Bus & $\begin{array}{c}\text { AC voltage } \\
\text { magnitude (p.u) }\end{array}$ & $\begin{array}{c}\text { AC voltage } \\
\text { angle(rad) }\end{array}$ & $\begin{array}{c}\text { DC voltage } \\
\text { of VSC }\end{array}$ \\
\hline 1 & 1.0000 & 0 & - \\
2 & 1.0018 & 0 & 1.0000 \\
3 & 0.9897 & -0.0383 & 1.0935 \\
4 & 0.9880 & -0.0431 & - \\
5 & 0.9892 & -0.0407 & 1.0926 \\
\hline
\end{tabular}

\section{Experimental validation}

The distributed OPF formulation for AC-DC grids has been tested in an experimental setup that runs the $3 \mathrm{DC}-5 \mathrm{AC}$ power system (analysed in steady state in Section 4) in real time through OPAL-RT (Figure 5). The goal of this implementation is to show the secure operation of the system under the distributed OPF guaranteeing minimum losses and adapting to load changes.

The data from the physical system is exchanged with the outside environment through an external switch and via user data protocol (UDP). A cluster of 5 Raspberry Pi (RPI 3, model B, 1 GB RAM, 1.2 GHz processor) is connected to the switch. Each Raspberry Pi is working as one of the agents of the system described in Section 4. Therefore, each Raspberry Pi is responsible for executing the OPF detailed in Algorithm 1, which has been implemented in Python for the experimental validation. The communication among agents is in a client/server manner and uses gRPC protocol. Each agent is a server that can receive incoming messages and dispatch them after a call and, at the same time, is a client of neighbour servers. The execution time of the solver in the different agents is depicted through boxplots in 
Figure $6 \mathrm{~b}$. It is shown that the median for solver execution in the different agents is between $0.4 \mathrm{~s}$ and $1 \mathrm{~s}$. The communication time between agents is represented through boxplots in Figure 6a. It is shown that the median of the communication time between the different agents is lower than 0.05 s. Even the power system under analysis was larger, the subsystems size will not change, so the computational costs per iteration will not be altered $[9,20]$. Furthermore, these results are very dependent on the characteristics of the Raspberry $\mathrm{Pi}$ used as agents. In a real environment, the computational capability of each agents would be larger, therefore the computational costs for the ADMM execution would be lower and this could empower the applicability of the proposed scheme in practical applications.

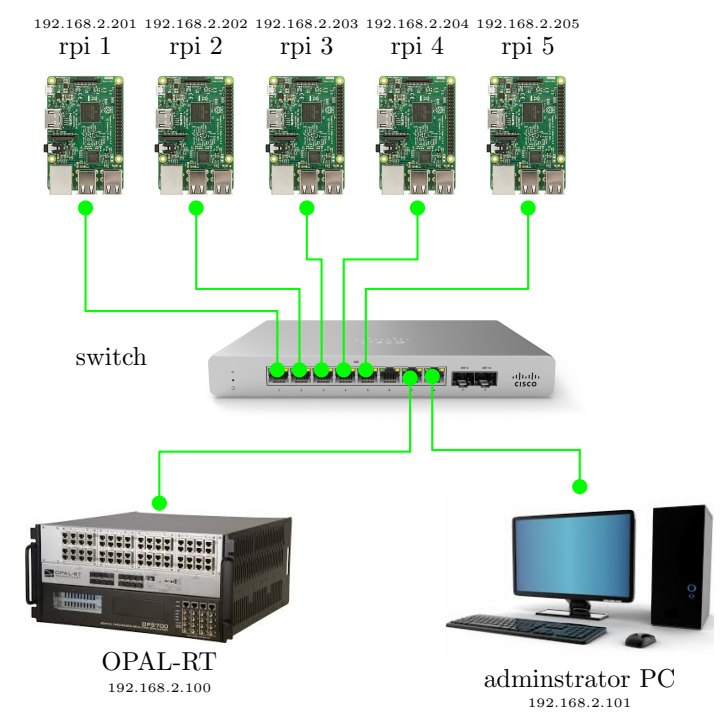

Figure 5: Experimental platform setup

The dynamic model of the system has been built in Matlab Simulink using the $\pi$ equivalent model for cables and representing VSC converters through its average model. Agent 1 is a special case because node 1 is the slack bus, therefore Agent 1 will not return setpoint results. For the tested scenario, it is assumed that the VSC connected to node DC 1 (VSC 1) works under DC voltage control-reactive power control mode, while VSCs connected to nodes DC2 (VSC 2) and DC3 (VSC 3) work under active power control-reactive power control mode. So, VSC 1 will receive a DC voltage and reactive power setpoint, which are determined by Agent 2. Agent 2 will also send the appropriate active and reactive power reference to the generator 


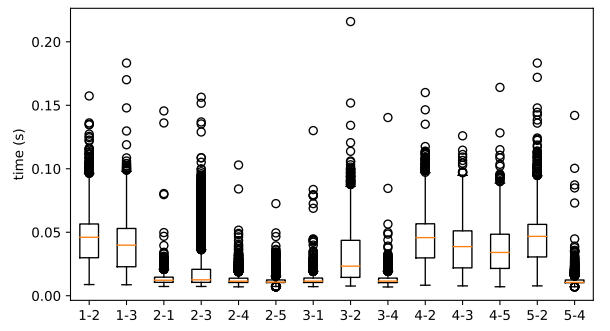

(a) Communication time between agents

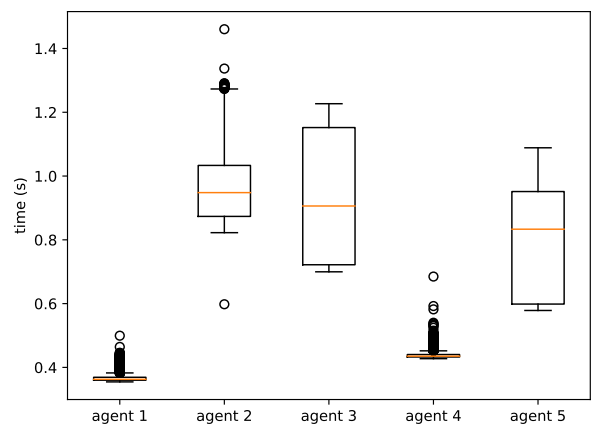

(b) Solver time in each agent

Figure 6: Communication time between agents and solver time in each agent

connected to AC node 2 . VSC 2 will receive an active power and a reactive power setpoint (determined by Agent 3) and VSC 3 will receive an active power and a reactive power setpoint (determined by Agent 5). The active and reactive power demanded in $\mathrm{AC}$ nodes $2,3,4$ and 5 are assumed to change in steps. The time instants when the load changes occur are marked in dark dash vertical lines in Figures $7 \mathrm{a}-7 \mathrm{~d}$. They happen at time instants $1802 \mathrm{~s}$ and $3602 \mathrm{~s}$ in $\mathrm{AC}$ nodes 2, 3, 4 and 5. The beginning and ending of each ADMM process is represented through pink dashed lines in Figures 7a-7d. So, it can be seen that for the test performed, 4 ADMM processes are executed. While each agent is performing the ADMM process, any demand change is perceived and taken into consideration for guiding the system to the optimal operation point that implies less system losses (Figure 8). The three steps happening at each agent of the system are the following:

(i) Each agent receives initial states which are local measurement of load active and reactive power from OPAL-RT lab through the interface. 
(ii) Based on local information from OPAL-RT and exchanged information from neighbour RPIs, each agent iteratively runs the ADMM process as described in Section 2. The number of iterations to guarantee the consensus in each ADMM process is 1100 for the $\rho=50.000$ that has been chosen.

(iii) At iteration 1100th, all agents finish an ADMM process loop. AC and $\mathrm{AC} / \mathrm{DC}$ agents send the optimal setpoints of active and reactive power to generators and VSCs that determine the new operational status. Then ADMM processes in the agents are restarted again from step i).
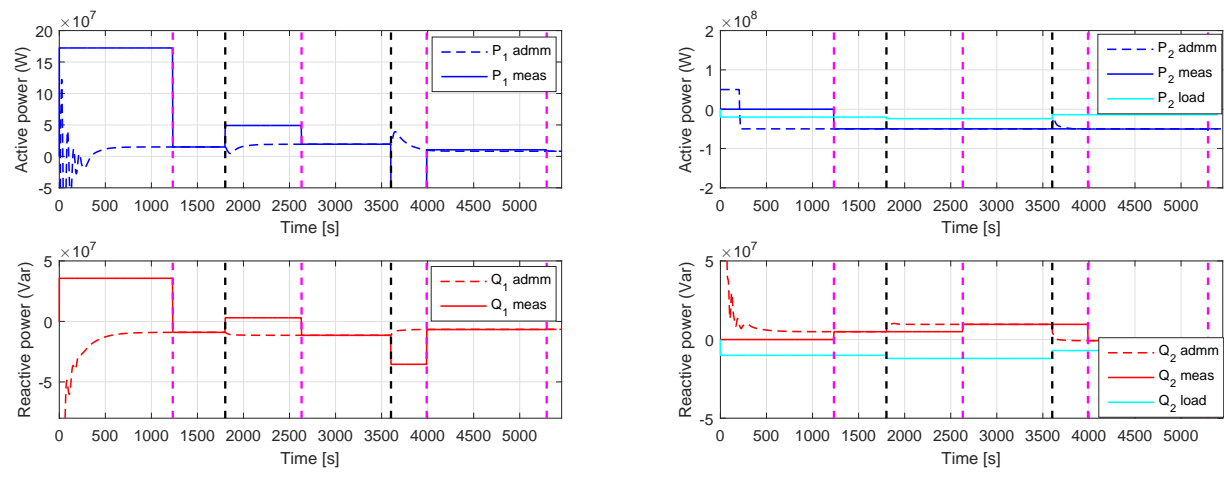

(a) Agent 1

(b) Agent 2
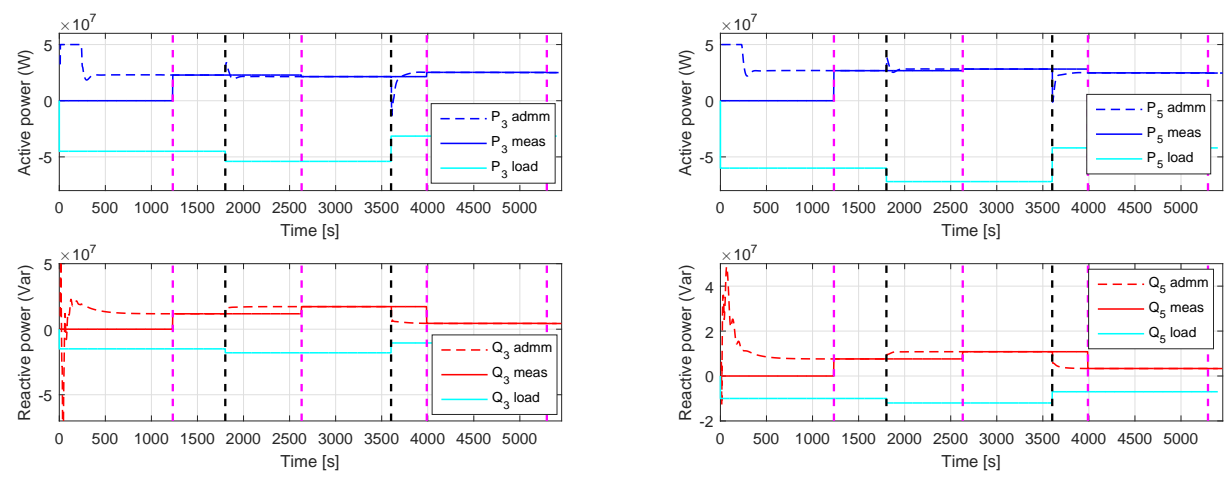

(c) Agent 3

(d) Agent 5

Figure 7: Active and reactive power in nodes $1,3,4$ and 5

In Figure 7, for each agent of buses 1, 3, 4 and 5, six signals are plotted (three for active power and three for reactive power). The measurement at 


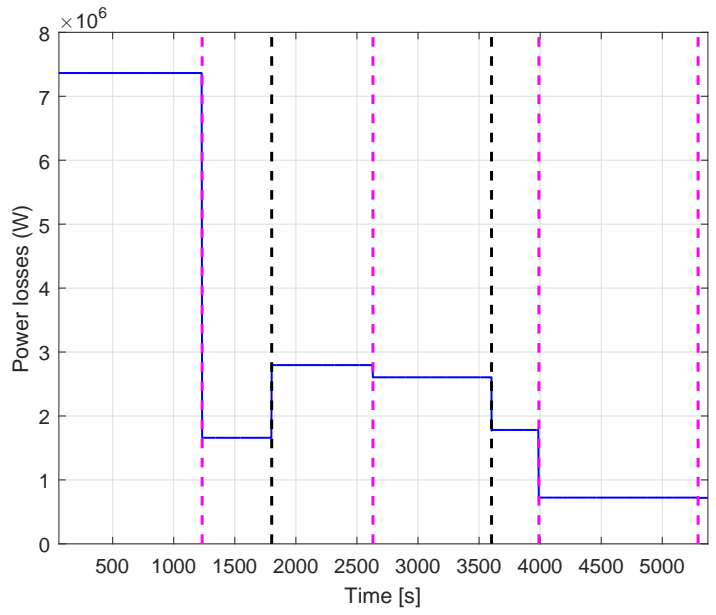

Figure 8: Power system losses
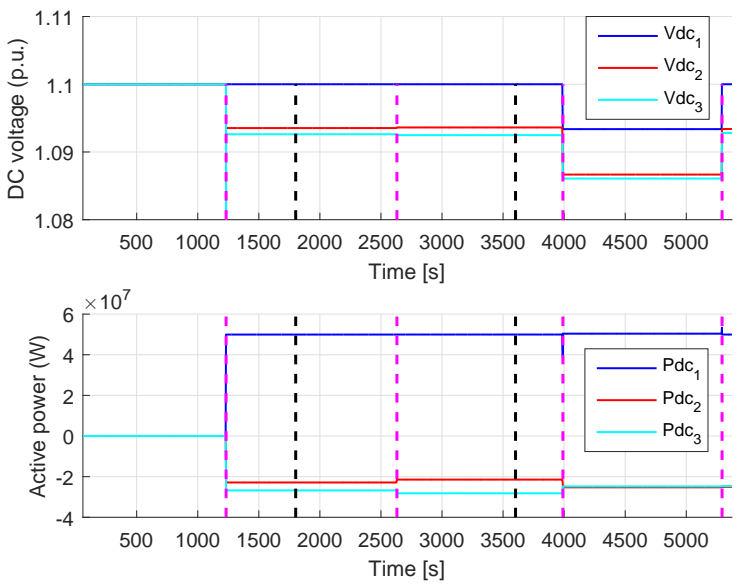

Figure 9: DC voltages and powers 
the node is represented by a continuous blue line for active power and continuous red line for reactive power. The demanded power (load) is represented by a cyan continuous line both for active and reactive power. The active power reference computed by the ADMM process is represented by a dashed blue line and the reactive power reference by a dashed red line. The active and reactive power references are not sent to the agent until the ADMM process has finished. When the ADMM process ends (which happens after 1100 iterations and is represented by the vertical pink dashed line), the power reference is sent to the agent and it can be seen that then the measurement is following the reference, until the demanded power changes or a new ADMM process starts. Once the ADMM running process has finished, a new ADMM process starts, to compute the new suitable reference for loss minimization according to the new demand. Figure 9 shows the voltages and powers of DC buses 1, 2 and 3, calculated by their corresponding agents. During the first ADMM process, when optimal results are not yet available, there is null power flow in the DC network (the three DC voltages have the same value). Once the ADMM setpoints are received, DC voltages are adapted to ensure optimal operation.

\section{Conclusions}

A distributed control for ensuring optimal operation in hybrid $\mathrm{DC} / \mathrm{AC}$ systems has been proposed. The developed formulation, based on ADMM and multi-agent systems, is designed to work as tertiary control in HVDCHVAC transmission systems for loss minimization. This scheme is an attractive alternative to centralized approaches: it only requires communications with immediate neighbour buses that allow voltage information exchange. It is shown that it reaches the same solution that the centralized approach in a 3 DC - 5 AC bus system. On the other hand, the proposed distributed control has been tested dynamically, in real time, after implementation in a laboratory setup. The system presents stable behaviour and adapts to demand changes, even if agents present different execution times for the ADMM process and different communication time between each other.

Taking into consideration the general formulation presented and the parallelization of computations of ADMM, the approach is scalable to larger power systems. It is worth noting that even if the power system is larger compared to the analysed one, the subsystems size will not change, so the computational costs per iteration will be in the same range $[9,20]$. The rela- 
tion between the admittance matrices of the AC and DC grids, as well as the penalty parameter have an important effect on the convergence speed and therefore on the computational costs. Further research could investigate the methodologies for the appropriate penalty parameter selection (which could be adaptive with the iteration number).

Last, but not least, recent studies suggest that Augmented Lagrangian Alternating Direction Inexact Newton (ALADIN) [16, 24] can reduce the number of iterations of ADMM, at the cost of an increased per-step communication effort. In [24] inexact Hessians are used to reduce the communication effort of ALADIN applied to distributed AC OPFs. Further research could investigate the suitability of ALADIN for distributed AC-DC OPFs.

\section{Acknowledgements}

Mònica Aragüés-Peñalba is Lecturer at UPC of the Serra Húnter Programme. This work was partially funded by FEDER / Ministerio de Ciencia, Innovación y Universidades - Agencia Estatal de Investigación Project RTI2018-095429-B-I00. This work has also been supported by the BD4OPEM H2020 project, which has received funding from the European Union's Horizon 2020 research and innovation program under Grant Agreement No. 872525.

\section{References}

[1] R. Teixeira Pinto, M. Aragüés-Peñalba, O. Gomis-Bellmunt, A. Sumper, Optimal operation of dc networks to support power system outage management, IEEE Transactions on Smart Grid 7 (6) (2016) 2953-2961. doi:10.1109/TSG.2016.2586024.

[2] Q. Nguyen, G. Todeschini, S. Santoso, Power flow in a multifrequency hvac and hvdc system: Formulation, solution, and validation, IEEE Transactions on Power Systems 34 (4) (2019) 2487-2497. doi:10.1109/TPWRS.2019.2896023.

[3] J. Beerten, R. Belmans, Development of an open source power flow software for high voltage direct current grids and hybrid ac/dc systems: Matacdc, IET Generation, Transmission Distribution 9 (10) (2015) 966974. 
[4] Z. Liang, S. Lin, M. Liu, Distributed optimal power flow of AC/DC interconnected power grid using synchronous ADMM, IOP Conference Series: Materials Science and Engineering 199 (2017) 012013. doi:10.1088/1757899x/199/1/012013.

[5] M. Aragüés-Peñalba, J. S. Bassols, S. G. Arellano, A. Sumper, O. G. Bellmunt, Optimal operation of hybrid high voltage direct current and alternating current networks based on opf combined with droop voltage control, International Journal of Electrical Power \& Energy Systems 101 (2018) 176 - 188. doi:https://doi.org/10.1016/j.ijepes.2018.03.010.

[6] H. Ergun, J. Dave, D. Van Hertem, F. Geth, Optimal power flow for ac/dc grids: Formulation, convex relaxation, linear approximation and implementation, IEEE Transactions on Power Systems (2019) 11doi:10.1109/TPWRS.2019.2897835.

[7] D. K. Molzahn, F. Dörfler, H. Sandberg, S. H. Low, S. Chakrabarti, R. Baldick, J. Lavaei, A survey of distributed optimization and control algorithms for electric power systems, IEEE Transactions on Smart Grid 8 (6) (2017) 2941-2962. doi:10.1109/TSG.2017.2720471.

[8] Y. Wang, S. Wang, L. Wu, Distributed optimization approaches for emerging power systems operation: A review, Electric Power Systems Research 144 (2017) 127 - 135.

[9] S. Boyd, N. Parikh, E. Chu, B. Peleato, J. Eckstein, Distributed optimization and statistical learning via the alternating direction method of multipliers, Foundations and Trends in Machine Learning 3 (1) (2011) $1-122$.

[10] T. Erseghe, Distributed optimal power flow using admm, IEEE Transactions on Power Systems 29 (5) (2014) 2370-2380.

[11] S. Magnússon, P. C. Weeraddana, C. Fischione, A distributed approach for the optimal power-flow problem based on admm and sequential convex approximations, IEEE Transactions on Control of Network Systems 2 (3) (2015) 238-253.

[12] Y. Wang, L. Wu, S. Wang, A fully-decentralized consensus-based admm approach for dc-opf with demand response, IEEE Transactions on Smart Grid 8 (6) (2017) 2637-2647. doi:10.1109/TSG.2016.2532467. 
[13] I. Khan, Z. Li, Y. Xu, W. Gu, Distributed control algorithm for optimal reactive power control in power grids, International Journal of Electrical Power \& Energy Systems 83 (2016) 505 - 513. doi:https://doi.org/10.1016/j.ijepes.2016.04.004.

[14] C. Gavriluta, R. Caire, A. Gomez-Exposito, N. Hadjsaid, A distributed approach for opf-based secondary control of mtdc systems, IEEE Transactions on Smart Grid 9 (4) (2018) 2843-2851.

[15] E. Iggland, R. Wiget, S. Chatzivasileiadis, G. Anderson, Multi-area dcopf for hvac and hvdc grids, IEEE Transactions on Power Systems 30 (5) (2015) 2450-2459. doi:10.1109/TPWRS.2014.2365724.

[16] N. Meyer-Huebner, M. Suriyah, T. Leibfried, Distributed optimal power flow in hybrid ac-dc grids, IEEE Transactions on Power Systems (2019) 1-1doi:10.1109/TPWRS.2019.2892240.

[17] S. Aziz, J. Peng, H. Wang, H. Jiang, Admm-based distributed optimization of hybrid mtdc-ac grid for determining smooth operation point, IEEE Access 7 (2019) 74238-74247. doi:10.1109/ACCESS.2019.2919700.

[18] Y. Li, Y. Li, G. Li, D. Zhao, C. Chen, Two-stage multi-objective opf for ac/dc grids with vsc-hvdc: Incorporating decisions analysis into optimization process, Energy 147 (2018) 286 - 296. doi:https://doi.org/10.1016/j.energy.2018.01.036.

[19] S. D. J. McArthur, E. M. Davidson, V. M. Catterson, A. L. Dimeas, N. D. Hatziargyriou, F. Ponci, T. Funabashi, Multi-agent systems for power engineering applications - part ii: Technologies, standards, and tools for building multi-agent systems, IEEE Transactions on Power Systems 22 (4) (2007) 1753-1759. doi:10.1109/TPWRS.2007.908472.

[20] T.-L. Nguyen, Agent-based distributed control and optimization in microgrids with hardware-in-the-loop implementation, Ph.D. thesis, Grenoble INP (2019).

[21] T.-L. Nguyen, E. Guillo-Sansano, M. Syed, V.-H. Nguyen, S. Blair, L. Reguera, Q.-T. Tran, R. Caire, G. Burt, C. Gavriluta, N.-A. Luu, Multi-agent system with plug and play feature for distributed secondary 
control in microgrid - controller and power hardware-in-the-loop implementation, Energies 11 (12).

[22] O. Anaya-Lara, J. O. Tande, K. Uhlen, M. K, Offshore Transmission Technology, John Wiley \& Sons, Ltd, 2018, Ch. 9, pp. 345-379. doi:10.1002/9781119097808.ch9.

[23] J. Beerten, S. Cole, R. Belmans, Generalized steady-state VSC MTDC model for sequential AC/DC power flow algorithms, IEEE Transactions on Power Systems 27 (2) (2012) 821-829. doi:10.1109/TPWRS.2011.2177867.

[24] A. Engelmann, Y. Jiang, T. Mühlpfordt, B. Houska, T. Faulwasser, Toward distributed opf using aladin, IEEE Transactions on Power Systems 34 (1) (2019) 584-594.

\section{Appendix}

\subsection{AC power flow formulation}

For an $\mathrm{AC}$ bus $k$, the injected apparent power power is given by $s_{k}$, with a real part corresponding to the injected active power $p_{k}$ and imaginary part corresponding to the injected reactive power $q_{k}$ :

$$
s_{k}=p_{k}+j q_{k}=\left\{\begin{array}{l}
s_{k}^{G}-s_{k}^{L} \text { if } k \in \mathcal{G}_{g e n} \\
-s_{k}^{L} \text { otherwise }
\end{array}\right.
$$

where $s_{k}^{G}$ and $s_{k}^{L}$ denote the apparent power of the generator and load, respectively, connected at bus $k$. The set of buses containing a generator is given by $\mathcal{G}_{\text {gen }}$. The injected power of all the buses is contained in vector $\mathbf{s}=\mathbf{p}+j \mathbf{q}$. The current injection at bus $k$ is defined as $i_{k}=i_{k}^{r e}+j i_{k}^{i m}$. The collection of the current injection in all the bus is contained in vector $\mathbf{i}=\mathbf{i}^{r e}+j \mathbf{i}^{i m}$. Similarly, the voltage of bus $k$ is $v_{k}=v_{k}^{r e}+j v_{k}^{i m}$ and the vector of all the system bus voltages $\mathbf{v}=\mathbf{v}^{r e}+j \mathbf{v}^{i m}$. The admittance value of line $(m, n)$ is $y_{m n}=g_{m n}+j b_{m n}$, where $g_{m n}$ represents the conductance and $b_{m n}$ represents the susceptance. The admittance of the AC network is expressed by matrix $\mathbf{Y}=\mathbf{G}+j \mathbf{B}$. Then, the relation between the bus voltages and the current injections can be expressed as follows:

$$
\mathbf{i}=\mathbf{Y} \cdot \mathbf{v}=\left(\mathbf{G} \cdot \mathbf{v}^{r e}-\mathbf{B} \cdot \mathbf{v}^{i m}\right)+j\left(\mathbf{B} \cdot \mathbf{v}^{r e}+\mathbf{G} \cdot \mathbf{v}^{i m}\right)
$$


The injected power can be expressed through voltages and current injections as:

$$
\mathbf{s}=\mathbf{v} \cdot \mathbf{i}^{*}=\left(\mathbf{v}^{r e} \cdot \mathbf{i}^{r e}+\mathbf{v}^{i m} \cdot \mathbf{i}^{i m}\right)+j\left(\mathbf{v}^{i m} \cdot \mathbf{i}^{r e}-\mathbf{v}^{r e} \cdot \mathbf{i}^{i m}\right)
$$

From Equation (30) and Equation (31), Equation (32) is obtained. Equation (33) results from separating the real and imaginary parts of Equation (32).

$$
\begin{gathered}
\mathbf{s}=\mathbf{p}+j \mathbf{q}=\mathbf{v} \cdot(\mathbf{Y} \cdot \mathbf{v})^{*} \\
\mathbf{p}=\mathbf{v}^{r e} \cdot\left(\mathbf{G} \cdot \mathbf{v}^{r e}-\mathbf{B} \cdot \mathbf{v}^{i m}\right)+\mathbf{v}^{i m} \cdot\left(\mathbf{B} \cdot \mathbf{v}^{r e}+\mathbf{G} \cdot \mathbf{v}^{i m}\right) \\
\mathbf{q}=\mathbf{v}^{i m} \cdot\left(\mathbf{G} \cdot \mathbf{v}^{r e}-\mathbf{B} \cdot \mathbf{v}^{i m}\right)-\mathbf{v}^{r e} \cdot\left(\mathbf{B} \cdot \mathbf{v}^{r e}+\mathbf{G} \cdot \mathbf{v}^{i m}\right)
\end{gathered}
$$

In order to simplify the previous expressions, the following notation will be used from now on:

$$
\begin{gathered}
\hat{\mathbf{v}}=\left[\begin{array}{c}
\mathbf{v}^{r e} \\
\mathbf{v}^{i m}
\end{array}\right] \\
\mathbf{z}^{p}=\left[\begin{array}{cc}
\mathbf{G} & -\mathbf{B} \\
\mathbf{B} & \mathbf{G}
\end{array}\right] \\
\mathbf{z}^{q}=\left[\begin{array}{cc}
-\mathbf{B} & -\mathbf{G} \\
\mathbf{G} & -\mathbf{B}
\end{array}\right]
\end{gathered}
$$

Then, the total injected active power and reactive power in the AC grid is given by Equations (38) and (39), respectively:

$$
\begin{aligned}
& \sum_{k=1}^{N} p_{k}=\hat{\mathbf{v}}^{T} \cdot \mathbf{z}^{p} \cdot \hat{\mathbf{v}} \\
& \sum_{k=1}^{N} q_{k}=\hat{\mathbf{v}}^{T} \cdot \mathbf{z}^{q} \cdot \hat{\mathbf{v}}
\end{aligned}
$$

The active power and reactive power balance at bus $k$ is determined by Equations (40) and (41):

$$
\begin{aligned}
p_{k} & =\hat{\mathbf{v}}_{k}^{T} \cdot \mathbf{z}_{k}^{p} \cdot \hat{\mathbf{v}}_{k} \\
q_{k} & =\hat{\mathbf{v}}_{k}^{T} \cdot \mathbf{z}_{k}^{q} \cdot \hat{\mathbf{v}}_{k}
\end{aligned}
$$

where vector $\hat{\mathbf{v}}_{k}$ and matrices $\mathbf{z}_{k}^{p}$ and $\mathbf{z}_{k}^{q}$ have the same size as $\hat{\mathbf{v}}, \mathbf{z}^{p}$ and $\mathbf{z}_{k}^{q}$, respectively and where all elements not involved in bus $k$ are replaced by 
zeros:

$$
\begin{aligned}
\mathbf{z}_{k}^{p}= & {\left[\begin{array}{c}
\mathbf{0} \\
\mathbf{z}^{p}(k,:) \\
\mathbf{0} \\
\mathbf{z}^{p}\left(k+N_{a c},:\right) \\
\mathbf{0}
\end{array}\right] } \\
\mathbf{z}_{k}^{q}= & {\left[\begin{array}{c}
\mathbf{0} \\
\mathbf{z}^{q}(k,:) \\
\mathbf{0} \\
\mathbf{z}^{q}\left(k+N_{a c},:\right) \\
\mathbf{0}
\end{array}\right] }
\end{aligned}
$$

where $\mathbf{z}^{p}(k,:)$ and $\mathbf{z}^{p}\left(k+N_{a c},:\right)$ correspond to row $k$ and row $k+N_{a c}$ of matrix $\mathbf{z}^{p}$, respectively and $\mathbf{0}$ is the zero matrix of the correct size. $\mathbf{z}_{k}^{q}$ is obtained similarly to matrix $\mathbf{z}_{k}^{p}$.

\subsection{AC-DC power flow formulation}

Assuming that some of the $\mathrm{AC}$ nodes will be connected to a $\mathrm{DC}$ grid through AC-DC converters, the voltage vector defined in Equation (35) and the matrices presented in Equation (36) and (37) can be rewritten as follows:

$$
\begin{array}{r}
\hat{\mathbf{v}}=\left[\begin{array}{c}
\mathbf{v}^{r e} \\
\mathbf{v}^{i m} \\
\mathbf{v}_{d c}
\end{array}\right] \\
\mathbf{z}^{p}=\left[\begin{array}{ccc}
\mathbf{G} & -\mathbf{B} & \mathbf{0} \\
\mathbf{B} & \mathbf{G} & \mathbf{0} \\
\mathbf{0} & \mathbf{0} & \mathbf{0}
\end{array}\right] \\
\mathbf{z}^{q}=\left[\begin{array}{ccc}
-\mathbf{B} & -\mathbf{G} & \mathbf{0} \\
\mathbf{G} & -\mathbf{B} & \mathbf{0} \\
\mathbf{0} & \mathbf{0} & \mathbf{0}
\end{array}\right]
\end{array}
$$

An additional matrix is required for the DC grid:

$$
\mathbf{z}^{d c}=\left[\begin{array}{ccc}
\mathbf{0} & \mathbf{0} & \mathbf{0} \\
\mathbf{0} & \mathbf{0} & \mathbf{0} \\
\mathbf{0} & \mathbf{0} & \mathbf{G}_{d c}
\end{array}\right]
$$


where $\mathbf{G}_{d c}$ corresponds to the admittance matrix of the DC grid. The dimension of vector $\hat{\mathbf{v}}$ is $\left(2 N_{a c}+N_{d c}\right) \times 1$ and the dimension of matrices $\mathbf{z}_{p}, \mathbf{z}_{q}$ and $\mathbf{z}_{d c}$ is $\left(2 N_{a c}+N_{d c}\right) \times\left(2 N_{a c}+N_{d c}\right)$. Equations (40), (41) are still valid for $\mathrm{AC}$ nodes and the construction of $\mathbf{z}_{k}^{p}$ (in Equation (42)) and $\mathbf{z}_{k}^{q}$ (in Equation (43)) also applies. For the DC nodes, the power can be expressed as:

$$
p_{l, d c}=\hat{\mathbf{v}}_{k}^{T} \cdot \mathbf{z}_{l}^{d c} \cdot \hat{\mathbf{v}}_{k}
$$

where $l$ represents the DC node connected to the $\mathrm{AC}$ node $k$. Matrix $\mathbf{z}_{l}^{d c}$ is computed as shown in Equation (49) knowing that $\mathbf{z}^{d c}\left(2 N_{a c}+l,:\right)$ corresponds to the row $2 N_{a c}+l$ of matrix $\mathbf{z}^{d c}$ and $\mathbf{0}$ is the zero matrix of the correct size.

$$
z_{l}^{d c}=\left[\begin{array}{c}
\mathbf{0} \\
\mathbf{z}^{d c}\left(2 N_{a c}+l,:\right) \\
\mathbf{0}
\end{array}\right]
$$

